\section{The impact of Kt/V urea-based dialysis adequacy on quality of life and adherence in haemodialysis patients: a cross-sectional study in Greece}

\section{Paraskevi Theofilou, 1 Constantinos \\ Togas,2 Chrysoula Vasilopoulou, 3 \\ Christos Minos, 4 Sofia Zyga, 5 \\ Giorgos Tzitzikos6}

1Sotiria Hospital for Thoracic Diseases, Athens; 2Social Worker-Psychologist;

3Department of Nurse Training, Continual

Renal Replacement Therapy, Ariti

Company, Acharnes; ${ }^{4}$ General Hospital of

Korinthos; ${ }^{5}$ Department of Nursing,

Faculty of Human Movement and Quality

of Life Sciences, University of

Peloponnese, Sparta; ${ }^{6}$ Renal Department,

General Hospital of Corinth, Greece

\section{Abstract}

There is clear evidence of a link between dialysis adequacy (as measured by urea kinetic modeling or urea reduction ratio) and such important clinical outcomes as morbidity and mortality. Evidence regarding the relationship between dialysis adequacy and quality of life (QOL) outcomes as well as adherence is less clear. The present paper is a study protocol which is planning to answer the following research question: what is the impact of dialysis adequacy on QOL and adherence in a sample of hemodialysis patients? The final sample size will be around 100 patients undergoing hemodialysis. Each subject's QOL and adherence will be measured using the following instruments: i) the Missoula-VITAS quality of life index 25; ii) the multidimensional scale of perceived social support and iii) the simplified medication adherence questionnaire. Dialysis adequacy is expected to be related to QOL and adherence scores.

\section{Background}

There are about 1.8 million patients with end-stage renal disease (ESRD) all over the world that need a kind of renal replacement therapy (RRT), including hemodialysis (HD), peritoneal dialysis (PD), or transplantation. ${ }^{1}$ Since the advent of dialysis, there have been many advances in the treatment of ESRD. However, mortality rates for ESRD patients remain several times higher than those of age matched controls without ESRD, ${ }^{2}$ and patients with ESRD continue to have significant impairments in health - related quality of life (HRQOL). 3,4

The factors that influence HRQOL in patients with ESRD have received little attention. Previous research has focused on the effects of erythropoietin, ${ }^{5}$ exercise, ${ }^{6-8}$ and on the impact of the various modalities of renal replacement therapy. ${ }^{9}$ Another factor that may influence HRQOL in ESRD patients is dialysis adequacy as evidenced by an uncontrolled study that showed an improvement in six of eight Short Form-36 (SF-36) domains in 14 patients whose $\mathrm{Kt} / \mathrm{V}$ levels increased on average by $0.2 .{ }^{10}$ Also, Mapes et al. ${ }^{11}$ have indicated the strong correlation between the risk of death as well as hospitalization in HD patients and HRQOL. Specifically, a 10-point lower physical component summary score was associated with higher elevation in the adjusted mortality risk, as was a $1 \mathrm{~g} / \mathrm{dL}$ lower serum albumin level. The effects of HD dose and membrane flux on HRQOL have been examined by Unruh et al.12 The HEMO Study results demonstrate the marked burden of chronic kidney failure and HD treatment on daily life. In this trial among patients undergoing maintenance three times a week hemodialysis, the SF-36 physical component summary score and pain scale showed significant but very small clinical effects favoring the higher dialysis dose. No clinically meaningful benefits or either the dose or flux interventions were observed for other indices of HRQOL.

In the present study, we are planning to use the Missoula-VITAS Quality of Life Index-25 (MVQ0LI-25), the Multidimensional Scale of Perceived Social Support (MSPSS), and the Simplified Medication Adherence Questionnaire (SMAQ) to determine whether dialysis adequacy is associated with HRQOL and adherence in a sample of HD patients.

\section{Methods}

A cohort of around one hundred patients undergoing HD treatment will be recruited from hospitals (public and private) located within the broader area of Athens and Peloponnese. The inclusion criteria are: i) $>18$ years of age; ii) ability of communication in Greek; iii) diagnosed with ESRD; iv) dialysis treatment at least for a year; v) satisfying level of cooperation and perceived ability; vi) no history of primary psychiatric disease that may interfere with conduct of study; vii) clinically stable with no evidence of chronic or acute infections, inflammatory disorders, malignancy. All subjects will be informed of their rights to refuse or discontinue participation in the
Correspondence: Paraskevi Theofilou, Sotiria Hospital for Thoracic Diseases, Athens; Center for Research and Technology, Department of Kinesiology, Health and Quality of Life Research Group, Trikala, Thessaly Eratous 12, 14568 , Athens, Greece.

Tel. $+30.697 .744 .1502,+30.210 .622 .1435$.

Fax +30.210 .622 .1435$

E-mail: theofi@otenet.gr; paraskevi.theofilou@ gmail.com

Key words: dialysis adequacy, hemodialysis, quality of life, adherence, social support.

Contributions: the authors contributed equally.

Conflict of interests: the authors declare no potential conflict of interests.

Received for publication: 9 February 2013.

Accepted for publication: 2 March 2013.

This work is licensed under a Creative Commons Attribution NonCommercial 3.0 License (CC BYNC 3.0).

(C) Copyright P. Theofilou et al., 2015

Licensee PAGEPress, Italy

Health Psychology Research 2015; 3:1060

doi:10.4081/hpr.2015.1060

study according to the ethical standards of the Helsinki Declaration. Ethical permission for the study will be obtained from the scientific committees of the participating hospitals.

The psychometric tools included in the study are presented below.

\section{Instruments}

Byock and Merriman created the MissoulaVITAS Quality of Life Index (MVQOLI). ${ }^{13}$ The MVQOLI is an assessment instrument that gathers patient-reported information about QOL during advanced illness. Maintaining optimal QOL is a core goal of palliative and hospice care, and information gathered via the MVQOLI assists health care professionals in identifying and addressing patient concerns that affect QOL.

The MVQOLI has been used in many different healthcare settings including hospice, hospital, home health, long-term care (including assisted living), outpatient palliative care, disease management and pre-hospice programs. 13 The framework of the MVQOLI is based on Ira Byock's work regarding growth and development at the end of life and the concepts of landmarks and tasks of life closure.13 The MVQOLI asks patients about 5 dimensions or domains of QOL: symptoms, function, interpersonal, well-being and transcendence. The instrument is specifically designed to assess the patients personal experience in each of these dimensions, hence the MVQOLI items 
are constructed with highly subjective language and no scores appear on the version of the tool seen by patients. The tool seeks to describe the qualitative and subjective experience of QOL in a way that can be quickly interpreted by professional caregivers. The scale has been translated and validated into Greek. ${ }^{14,15}$ MSPSS, which consists of 12 items, was developed by Zimet et al. ${ }^{16}$ to identify the social support factors perceived by the individuals. The scale is comprised of 3 groups depending on the source of support, each group consisting of 4 items. These are family $[3,4,8,11]$, friends $[6,7,9,12]$ and a special person $[1,2,5,10]$. Each item is rated using a 7 range scale varying between definitely no and definitely yes. The sum of 4 items under each sub-scale gives the sub-scale score, while the sum of all sub-scale scores gives the overall scale score. The lowest score in sub-scales is 4 , and the highest is 28 . The lowest overall scale score is 12 , and the highest is 84 . MSPSS has proven to be psychometrically sound in diverse samples and to have good internal reliability and test-retest reliability, and robust factorial validity. 16,17 Specifically, in terms of reliability, the internal consistencies of the total scale and the sub-scales are high, ranging from 0.79 to 0.98 in various samples; furthermore, the test-retest reliability over a 2 to 3 -month period produces correlations ranging from 0.72 to 0.85 .16 The scale has been translated and validated into Greek. 18,19

The SMAQ questionnaire was developed as a modification of the Morisky-Green questionnaire to measure adherence to antiretroviral treatment in patients with acquired immunodeficiency syndrome (AIDS). This questionnaire consists of six questions that evaluate different aspects of patient compliance with treatment: forgetfulness, routine, adverse effects, and a quantification of omissions.$^{20} \mathrm{~A}$ patient is classified as non-compliant if he/she responds to any of the questions with a nonadherence answer, and in terms of quantification, if the patient has lost more than two doses during the last week or has not taken medication during more than two complete days during the last three months. This questionnaire was validated in a sample of Spanish patients under treatment with unboosted nelfinavir between 1998 and 1999.20 The present questionnaire has been translated into Greek and is under validation. ${ }^{21}$

The clinical characteristics of all patients, including demographic and laboratory data, like HD adequacy $(\mathrm{Kt} / \mathrm{V})$ and type of HD treatment (bicarbonate dialysis or haemodiafiltration) will be collected as baseline information at the beginning of the study.

\section{Conclusions}

The aim of the present study is to examine the relation between dialysis adequacy and QOL as well as adherence in a group of HD patients. We believe that the findings will contribute to the scientific community demonstrating the importance of laboratory data in the evaluation of HD patients' QOL and adherence rates.

The results will guide medical staff in individualizing the medical plan for HD patients. We conclude that a stepwise HD program is essential for this group of patients. This gradual approach to HD prescription need not be at the expense of QOL in HD patients.

\section{References}

1. Grassmann A, Gioberge S, Moeller S. Brown G. ESRD patients in 2004: global overview of patient numbers, treatment modalities and associated trends. Nephrol Dial Transplant 2005;20:2587-93.

2. Harnett JD, Kent GM, Foley RN, Parfrey PS. Cardiac function and hematocrit level. Am J Kidney Dis 1995;25:3-7.

3. Theofilou P. Self-reported functional status: an important predictor of mental health outcomes among chronic dialysis patients. Eur J Psychological Assess 2013;29:276-82.

4. Theofilou P. Quality of life and mental health in hemodialysis and peritoneal dialysis patients: the role of health beliefs. Int Urol Nephrol 2012;44:245-53.

5. Canadian Erythropoietin Study Group. Association between recombinant human erythropoietin and quality of life and exercise capacity of patients receiving haemodialysis. BMJ 1990;300:573-8.

6. Tawney KW, Tawney PJ, Hladik G. The life readiness program: a physical rehabilitation program for patients on hemodialysis. Am J Kidney Dis 2000;36:581-91.

7. Kutner NG, Zhang R, McClellan WM. Patient-reported quality of life early in dialysis treatment: Effects associated with usual exercise activity. Nephrol Nurs J 2000;27:357-67.

8. Theofilou P. Evaluation of quality of life in Greek patients with heart failure: the role of health cognitions before and after participation in an exercised-based rehabilitation program. J Clin Trials 2012:2:1-5.

9. Laupacis A, Keown P, Pus N. A study of the quality of life and cost-utility of renal transplantation. Kidney Int 1996;50:23542.
10. Powers KM, Wilkowski MJ, Helmandollar $\mathrm{AW}$, et al. Improved urea reduction ratio and $\mathrm{Kt} / \mathrm{V}$ in large hemodialysis patients using two dialyzers in parallel. Am J Kidney Dis 2000;35:266-74.

11. Mapes DL, Lopes AA, Satayathan S, et al. Health-related quality of life as a predictor of mortality and hospitalization: the Dialysis Outcomes and Practice Patterns Study (DOPPS). Kidney Int 2003;64:33949.

12. Unruh M, Benz R, Greene T. et al. Effects of hemodialysis dose and membrane flux on health-related quality of life in the HEMO Study. Kidney Int 2004;66:355-66.

13. Schwartz CE, Merriman M, Reed G, et al. Evaluation of the Missoula-VITAS quality of life index-revised: research tool or clinical tool? J Pall Med 2005;8:121-35.

14. Theofilou P, Kapsalis F, Panagiotaki H. Greek version of MVQOLI-15: translation and cultural adaptation. Int $\mathrm{J}$ Caring Sci 2012;5:289-94.

15. Theofilou P, Aroni A, Ralli M, et al. Measuring health-related quality of life in hemodialysis patients: psychometric properties of the Missoula-VITAS quality of life index (MVQOLI-15) in Greece. Health Psychol Res [In press].

16. Eker D, Arkar H, Yaldiz H. Generality of support sources and psychometric properties of a scale of perceived social support in Turkey. Soc Psychiatry Psychiatr Epidemiol 2000;35:228-33.

17. Zimet G, Dahlem N, Zimet S, Farley G. The Multidimensional scale of perceived social support. J Pers Assess 1988;52:30-41.

18. Theofilou P. The relation of social support to mental health and locus of control in chronic kidney disease. J Renal Nurs 2012;4:18-22.

19. Theofilou P, Zyga S, Tzitzikos G, et al. Assessing social support in Greek patients on maintenance hemodialysis: psychometric properties of the Multidimensional Scale of Perceived Social Support". In: Chronic kidney disease: signs/symptoms, management options and potential complications. New York: Nova Publishers; 2013. pp 265-279.

20. Ortega Suárez FJ, Sánchez Plumed J, Pérez Valentín MA, et al. Validation on the simplified medication adherence questionnaire (SMAQ) in renal transplant patients on tacrolimus. Nefrología 2011;31:690-6.

21. Theofilou P. Results from the translation and cultural adaptation of the Greek simplified medication adherence questionnaire (GR-SMAQ) in patients with lung cancer. J Clin Trials 2012;1:1-3. 